\section{Fatores familiares associados à obesidade abdominal entre adolescentes}

\section{Family factors associated with abdominal obesity in adolescents}

\section{Abstract}

Objectives: to investigate the association between family risk factors and abdominal obesity in adolescents.

Methods: the sample comprised 691 young people of both sexes (aged between 11 and 17 years), residing in the municipality of Presidente Prudente, in the State of São Paulo, in 2007. Identification of abdominal obesity was based on the waist circumference and family risk factors (level of schooling and overweight in the parents, socio-economic conditions, number of siblings and number of televisions in the household). These variables were collected using questionnaires. Statistical analysis involved application of the chi-squared test and logistic regression (odds ratio [OR] and confidence interval [C195\%]).

Results: the prevalence of abdominal obesity was $14.8 \%$ in the study sample and there was no difference between males and females (male: $17.7 \%$ and female: 12.9\%; $p=0.111)$. Abdominal obesity was associated with the private school sector (3.75 [1.27-11.00]), an overweight/obese mother (3.82 [1.14-12.73]) and both parents being overweight/obese (6.21 [2.0718.63])

Conclusions: the findings of this study suggest that studying in a private school and overweight parents are the main risk factors associated with abdominal obesity in adolescents.

Key words Abdominal circumference, Risk factors, Obesity, Adolescents
Rômulo Araújo Fernandes 1

Juliano Casonatto 2

Diego Giuliano Destro Christofaro 3

Gabriel Grizzo Cucato 4

Arli Ramos de Oliveira 5

Ismael Forte Freitas Júnior 6

\author{
Departamento de Educação Física. Instituto de Biociências. \\ Universidade Estadual Paulista (UNESP). Av. 24-A, 1515. \\ CEP: 13.500-900. Rio Claro, SP, Brasil. \\ E-mail: romulo_ef@yahoo.com.br \\ 2-5 Centro de Educação Física e Esportes. Universidade Estadual \\ de Londrina (UEL). Londrina, PR, Brasil. \\ 6 Departamento de Educação Física. Universidade Estadual \\ Paulista (UNESP). Presidente Prudente, SP, Brasil.
}

\section{Resumo}

Objetivos: analisar a associação entre fatores de risco familiares e a presença de obesidade abdominal entre adolescentes.

Métodos: a amostra foi composta por 691 jovens de ambos os gêneros (11 a 17 anos), residentes em Presidente Prudente-SP no ano de 2007. A identificação da obesidade abdominal foi baseada no valor da circunferência de cintura, e os fatores de risco familiares (escolaridade e excesso de peso dos pais, condição socioeconômica, número de irmãos e quantidade de televisores na residência) foram analisados por meio de questionários. A análise estatística utilizou o teste qui-quadrado e regressão logística (razão de chance [RC] e intervalos de confiança [IC95\%]).

Resultados: a prevalência de obesidade abdominal foi igual a 14,8\% na amostra avaliada e não foi diferente entre os gêneros (masculino: 17,7\% e feminino: $12,9 \%$; $p=0,111)$. A obesidade abdominal associou-se com a rede privada de ensino $(3,75$ [1,2711,00]), sobrepeso/obesidade da mãe $(3,82$ [1,1412,73]) e de ambos os pais (6,21 [2,07-18,63]).

Conclusões: os achados do presente estudo indicam que estudar na rede privada de ensino e o excesso de peso dos pais caracterizam importantes fatores de risco associados à presença da obesidade abdominal entre adolescentes.

Palavras-chave Circunferência abdominal, Fatores de risco, Obesidade, Adolescentes 


\section{Introdução}

Entre populações jovens, informações provenientes de estudos longitudinais têm evidenciado um aumento significativo na ocorrência do sobrepeso/ obesidade $(\mathrm{S} / \mathrm{O})$, constituindo assim uma importante fonte de preocupação para profissionais da área da saúde.1,2 Essa preocupação alicerça-se no fato de que tal morbidade está associada a um aumento do risco de desenvolvimento de doenças cardiovasculares já durante a infância e a adolescência. ${ }^{3-5}$

A obesidade abdominal ou central tem sido foco de diversas investigações, uma vez que a gordura acumulada nessa região do tronco possui taxas de lipólise mais altas e, consequentemente, associa-se com os diferentes componentes da síndrome metabólica.5,6 Dessa forma, é considerada melhor indicador de riscos para a saúde do que a obesidade geral.

No que se refere às características de diagnóstico, a circunferência de cintura (CC) tem se apresentado como um indicador simples, de fácil aplicabilidade e eficiente para a detecção da obesidade abdominal e também do risco cardiovascular.7,8 Entretanto, existe uma escassez de informações referentes à ocorrência desse fenômeno entre adolescentes brasileiros, bem como dos possíveis fatores de risco associados a ele.

Nesse sentido, evidências da literatura têm apontado que a obesidade geral é um fenômeno de múltiplas origens e substancialmente influenciado por fatores genéticos e ambientais. Todavia, independentemente dos diferentes fatores genéticos ou ambientais associados ao desenvolvimento da obesidade, é consenso na literatura que o núcleo familiar é um agente em potencial associado a esse processo.9-13 Assim, fatores familiares relacionados ao convívio com os hábitos paternos/maternos (excesso de peso dos pais e maior escolaridade dos mesmos - este segundo podendo até ser tratado como agente protetor), maior concentração de renda/disponibilidade de alimentos para o jovem em questão (menor número de pessoas na residência e melhor condição econômica) e maior prática de comportamentos sedentários em casa (número de televisores na residência), têm sido frequentemente associados ao excesso de peso, mas pouco investigados em relação à obesidade abdominal.9-13

Portanto, em decorrência da ausência de informações provenientes da associação entre obesidade abdominal e fatores de risco, o objetivo do presente estudo foi analisar a ocorrência da mesma entre adolescentes de ambos os gêneros, bem como as possíveis associações com diferentes fatores de risco familiares.

\section{Métodos}

A presente pesquisa é descritiva/analítica, de delineamento transversal, e foi conduzida na cidade de Presidente Prudente (IDH=0,846), a maior na região oeste do Estado de São Paulo, durante o segundo semestre de 2007. O tamanho da amostra de 556 sujeitos foi calculado para detectar uma prevalência de obesidade abdominal de $15,4 \%, 14$ com um erro amostral de $3 \%$, poder de $80 \%$ e significância estatística de $5 \%$. Com base em dados de um estudo anterior, onde foi observada uma perda amostral de $5,6 \%$, planejou-se a coleta de informações referentes a no mínimo 588 sujeitos.

Para a seleção da amostra, durante a primeira fase da pesquisa, dados foram levantados e, do total de escolas da cidade $(\mathrm{n}=117)$, foram listadas aquelas que atendiam $(\mathrm{n}=36)$ à faixa etária envolvida no estudo (11-17 anos). Durante a segunda fase do processo de seleção, entre as 36 unidades escolares previamente selecionadas (respeitando a proporcionalidade das redes de ensino), três públicas e uma privada foram escolhidas aleatoriamente para participar da pesquisa.

Nas unidades escolares selecionadas, todos os adolescentes matriculados nos ensinos fundamental e médio foram convidados a participar (1200 alunos elegíveis). Desses, 721 concordaram em participar, declararam não possuir nenhuma doença metabólica diagnosticada e retornaram com o termo de consentimento assinado pelos responsáveis. Porém, 30 escolares foram excluídos da análise por apresentar alguma irregularidade em suas informações (questionários em branco/preenchidos de maneira inadequada; ausência nos dias de avaliação). Dessa forma, a amostra foi composta por 691 (58\%) adolescentes de ambos os sexos e idades variando de 11 a 17 anos (masculino: 266 e feminino: 425). O protocolo de estudo foi aprovado pelo Comitê de Ética em Pesquisa da UNESP de Presidente Prudente (Processo no. 087/2006).

Todas as avaliações foram realizadas em salas nas escolas, durante as aulas de Educação Física. A idade cronológica dos adolescentes foi determinada em forma centesimal utilizando a data de nascimento e o dia da avaliação. O peso corporal foi aferido com a utilização de uma balança portátil digital (graduação: $100 \mathrm{~g}$ e capacidade máxima: $150 \mathrm{~kg}$ ) e a estatura com um estadiômetro de metal fixado à parede (precisão: $0,1 \mathrm{~cm}$ e extensão máxima: dois metros), seguindo metodologia padrão.15 Adicionalmente, calculou-se o índice de massa corporal (IMC 
$\left.\left[\mathrm{kg} / \mathrm{m}^{2}\right]\right)$. O valor da circunferência de cintura (CC) foi tomado em duplicata, seguindo metodologia padrão, 16 e os valores críticos elaborados por Taylor et al. 17 indicaram a obesidade abdominal.

Todos os fatores de risco familiares foram selecionados com base em relatos da literatura, onde os mesmos são apontados como agentes associados ao excesso de peso. ${ }^{9-13}$ Nesse sentido, foram considerados: maior escolaridade e excesso de peso dos pais, maior condição socioeconômica (CSE), número de filhos (um único filho), número de aparelhos televisores na residência ( $\geq 2$ aparelhos) e âmbito escolar (escola particular). Todos os adolescentes preencheram, com o auxílio de avaliadores treinados, um questionário para CSE. 18 Com base nas informações fornecidas por esse instrumento, os adolescentes foram classificados em sete possíveis grupos para $\mathrm{CSE}\left(\mathrm{A}^{1}, \mathrm{~A}^{2}, \mathrm{~B}^{1}, \mathrm{~B}^{2}, \mathrm{C}, \mathrm{D}\right.$ e E), sendo considerado fator de risco estar situado nos quatro grupos mais altos $\left(\mathrm{A}^{1}\right.$, $\left.\mathrm{A}^{2}, \mathrm{~B}^{1}, \mathrm{~B}^{2}\right)$. O número de aparelhos televisores foi avaliado por meio do mesmo instrumento e considerou-se fator de risco uma quantidade igual ou superior a dois aparelhos.

Um segundo questionário (preenchido pelos pais/responsáveis no próprio domicílio) foi entregue aos adolescentes e recolhido no dia seguinte. Esse instrumento possuía questões sobre a escolaridade dos pais, número de irmãos do adolescente em questão (morando na mesma residência) e solicitava que fossem reportados valores de peso corporal e estatura. Foram considerados fatores de risco: possuir curso superior completo, possuir um único filho (o adolescente em questão) e excesso de peso dos pais (IMC $\geq 25 \mathrm{~kg} / \mathrm{m}^{2}$ ). Uma semana após o recolhimento do instrumento, trinta pais (15 pais e 15 mães) foram selecionados aleatoriamente e convidados a comparecer nas escolas para novo preenchimento de questionário e realização de uma avaliação antropométrica. Os valores de concordância foram altos $(\mathrm{k}=1,0$ para escolaridade e número de filhos; $k=0,85$ para estado nutricional [IMC $\geq 25 \mathrm{~kg} / \mathrm{m}^{2}$ ]).

$\mathrm{O}$ teste de Komolgorov-Smirnov $(K-S)$ indicou a necessidade de utilizar estatística não-paramétrica para os dados numéricos. A estatística descritiva foi composta por mediana e diferença entre o percentil $75(\mathrm{Q} 3)$ e $25(\mathrm{Q} 1)$ e o teste $U$ de Mann-whitney estabeleceu comparações entre os gêneros. O teste quiquadrado analisou a existência de associações entre os fatores de risco familiares (variáveis independentes) e a presença da obesidade abdominal (variável desfecho), e a regressão logística binária (razão de chance [RC] e intervalo de confiança de 95\% [IC95\%]) indicou a magnitude dessas associações. Adicionalmente, foi criado um modelo ajus- tado para a regressão logística, onde foram inseridas apenas as variáveis independentes que apresentam significância estatística no modelo univariado. O índice kappa $(k)$ analisou a concordância dos dados em ambos os momentos avaliados (reprodutibilidade). O software SPSS versão 13.0 (Statistical Package for Social Science, SPSS Inc, Illinois, USA) foi utilizado e o nível de significância adotado foi de $p<0,05$.

\section{Resultados}

Do total de 1200 alunos, um universo de 479 não participou no estudo: 89 recusaram-se a receber o termo de consentimento, 225 não obtiveram a permissão de seus responsáveis, 72 acusaram a "perda" do termo de consentimento e 93 recusaramse a participar das avaliações antropométricas no dia marcado. Na Tabela 1 são apresentados os valores medianos referentes às características gerais da amostra analisada. O grupo do sexo feminino representou aproximadamente $60 \%$ do total de indivíduos envolvidos no estudo. Entre os gêneros, no que se refere às variáveis antropométricas, não houve diferença significativa do ponto de vista estatístico para as variáveis idade e IMC.

Em toda a amostra, 14,8\% dos adolescentes apresentaram obesidade abdominal, não sendo possível observar associação com o gênero (masculino: $17,7 \%$ e feminino: $12,9 \% ; p=0,111$ ). $\mathrm{O}$ teste qui-quadrado apontou a existência de associação significativa entre obesidade abdominal e os fatores de risco familiares: $\operatorname{CSE}(p=0,008)$, âmbito escolar $(p=0,018)$, excesso de peso paterno $(p=0,048)$, materno $(p=0,026)$ e de ambos os pais $(p=0,001)$.

A regressão logística indicou para as associações significativas no modelo univariado (teste quiquadrado), a magnitude das mesmas. Na Tabela 2 também é apresentado um modelo ajustado com os valores de RC para todas as variáveis analisadas. Com exceção das variáveis $\operatorname{CSE}(p=0,076)$ e excesso de peso paterno $(p=0,074)$, todas as demais mantiveram associação significativa com a presença da obesidade abdominal no grupo de adolescentes analisado. 
Tabela 1

Características gerais da amostra. Presidente Prudente, São Paulo. ( $N=691)$.

\begin{tabular}{|c|c|c|c|c|}
\hline \multirow[t]{2}{*}{ Variáveis } & \multicolumn{2}{|c|}{ Masculino $(\mathrm{N}=266)$} & \multicolumn{2}{|c|}{ Feminino $(\mathrm{N}=425)$} \\
\hline & Md & $\begin{array}{l}\text { Diferenças } \\
\text { entre } \\
\text { percentil } \\
75 \text { e } 25\end{array}$ & Md & $\begin{array}{c}\text { Diferenças } \\
\text { entre } \\
\text { percentil } \\
75 \text { e } 25\end{array}$ \\
\hline Idade (anos) & 13,8 & 3,1 & 13,6 & 2,1 \\
\hline Peso corporal (kg) & 54,7 & 20,9 & 49,1 & 12,8 \\
\hline Estatura $(\mathrm{m})$ & 1,65 & 0,6 & 1,59 & 0,1 \\
\hline IMC (kg/m²) & 19,6 & 5,1 & 19,4 & 4,2 \\
\hline $\mathrm{CC}(\mathrm{cm})$ & 69,8 & 12,1 & 66,1 & 10,8 \\
\hline
\end{tabular}

$C C=$ ircunferência de cintura; IMC=índice de massa corporal; Md=Mediana.

Tabela 2

\begin{tabular}{|c|c|c|c|c|c|}
\hline \multirow[t]{2}{*}{ Fatores de risco } & \multirow[t]{2}{*}{$\begin{array}{l}\text { Examinados } \\
\text { (N) }\end{array}$} & \multicolumn{2}{|c|}{$\begin{array}{l}\text { Obesidade } \\
\text { abdominal }\end{array}$} & \multicolumn{2}{|c|}{ Odds Ratio } \\
\hline & & $\mathrm{n}$ & $\%$ & OR bruta (IC95\%) & OR ajustada (IC95\%) \\
\hline \multicolumn{6}{|c|}{ Condição socioeconômica } \\
\hline Baixa & 232 & 22 & 9,5 & 1,00 & 1,00 \\
\hline Alta & 459 & 80 & 17,4 & $2,01(1,22-3,32)$ & $1,89(0,93-3,83)$ \\
\hline \multicolumn{6}{|l|}{ Tipo de escola } \\
\hline Pública & 591 & 82 & 13,8 & 1,00 & 1,00 \\
\hline Privada & 100 & 27 & 27,0 & $2,30(1,20-4,43)$ & $3,75(1,27-11,0)$ \\
\hline \multicolumn{6}{|l|}{ Número de irmãos } \\
\hline$\geq 1$ irmão & 635 & 89 & 14,1 & 1,00 & 1,00 \\
\hline Nenhum & 56 & 10 & 17,9 & $1,32(0,64-2,71)$ & $1,30(0,60-2,70)$ \\
\hline \multicolumn{6}{|l|}{ Escolaridade Pai } \\
\hline Sem nível superior & 600 & 88 & 14,7 & 1,00 & 1,00 \\
\hline Com nível superior & 91 & 14 & 15,4 & $1,05(0,57-1,95)$ & $1,04(0,56-1,94)$ \\
\hline \multicolumn{6}{|l|}{ Escolaridade Mãe } \\
\hline Sem nível superior & 599 & 86 & 14,4 & 1,00 & 1,00 \\
\hline Com nível superior & 92 & 16 & 17,4 & $1,25(0,69-2,25)$ & $1,23(0,69-2,23)$ \\
\hline \multicolumn{6}{|l|}{ Número de TV } \\
\hline TV $(0 / 1)$ & 205 & 28 & 13,7 & 1,00 & 1,00 \\
\hline 2-3 TV & 429 & 61 & 14,2 & $1,04(0,64-1,69)$ & $1,04(0,64-1,68)$ \\
\hline$\geq 4 \mathrm{TV}$ & 57 & 13 & 22,8 & $1,86(0,89-3,89)$ & $1,86(0,89-3,90)$ \\
\hline \multicolumn{6}{|c|}{ Sobrepeso/obesidade dos pais } \\
\hline Nenhum & 377 & 18 & 4,8 & 1,00 & 1,00 \\
\hline Pai & 126 & 17 & 13,5 & $3,11 \quad(1,01-9,62)$ & $3,81(0,90-8,75)$ \\
\hline Mãe & 75 & 12 & 16,1 & $3,81(1,17-12,38)$ & $3,82(1,14-12,7)$ \\
\hline Ambos & 113 & 28 & 24,8 & $6,58(2,21-19,61)$ & $6,21(2,07-18,6)$ \\
\hline
\end{tabular}

IC95\%=intervalo de confiança de $95 \%$; TV=aparelho televisor. 


\section{Discussão}

Com relação aos adolescentes brasileiros, informações referentes à ocorrência da obesidade abdominal são escassas, fato que limita comparações com dados de outras regiões do país. Nesse sentido, dados recentes (utilizando os mesmos pontos de corte) entre adolescentes da rede privada de ensino do mesmo município analisado indicaram uma elevada ocorrência de obesidade abdominal $(15,4 \%) .14$ Os valores são próximos aos 14,8\% observados nessa amostra composta por adolescentes de ambas as redes de ensino e evidenciam a relevância do problema entre os escolares de presidente Prudente, independentemente de a escola ser pública ou privada.

Ainda abordando o âmbito escolar, para a presente amostra, estudar em escola particular apresentou-se como um importante fator de risco associado à presença da obesidade abdominal. Em diferentes regiões do país, diversos estudos que utilizaram indicadores de obesidade geral, como é o caso do IMC, já reportaram esta associação entre crianças e adolescentes.19-21 Uma possível justificativa para isso foi observada por Nunes et al.,22 que identificaram um maior consumo por parte dos adolescentes da rede privada de ensino, dentro da própria unidade escolar, de alimentos ricos em gorduras saturadas, tais como salgadinhos, frituras e refrigerantes.

Nesse sentido, com base também em informações indicando que ações bem conduzidas em meio escolar podem diminuir de forma significativa a prevalência da obesidade e a adoção de hábitos de risco à saúde entre escolares, 23 cabe a orientação de que, dependendo das oportunidades oferecidas aos seus alunos, o meio escolar pode caracterizar-se como um importante agente tanto de combate como de promoção ao desenvolvimento da obesidade.

Paralelamente, uma maior CSE, que em países em processo de industrialização associa-se com fatores de risco relacionados ao desenvolvimento do S/O (maior tempo despendido assistindo televisão) ${ }^{24}$ e com a própria ocorrência do S/O entre adolescentes, 1,22 associou-se com a obesidade abdominal apenas no modelo univariado, perdendo sua significância estatística no modelo final. No presente estudo, os resultados indicam que essa associação da CSE com a obesidade abdominal aparentemente pode ser mediada por fatores como o meio escolar e o estado nutricional dos pais. Corroborando com tais dados, em investigação anterior, Fernandes et al. 25 demonstraram que o S/O associa-se de diferentes maneiras com o mesmo fator risco dependendo do estrato econômico onde é analisado e, o único fator de risco que se manteve associado ao $\mathrm{S} / \mathrm{O}$, independentemente da CSE, foi o $\mathrm{S} / \mathrm{O}$ dos pais.

Portanto, a forte relação existente entre o excesso de peso dos pais e de seus filhos tem sido frequentemente verificada em estudos anteriores, ${ }^{9-13}$ sendo essa relação observada para a obesidade abdominal no presente estudo e também entre adolescentes suecos. ${ }^{26}$ Entretanto, a literatura não apresenta de forma concisa uma definição sobre o real impacto de fatores genéticos e ambientais nessa relação. Nesse sentido, o fato de o excesso de peso corporal paterno ter perdido significância no modelo final de regressão, enquanto o materno se manteve significativo, além de estar em concordância com outras informações da literatura para excesso de peso 13 e obesidade abdominal 25 aponta para importante papel de fatores ambientais nesse processo, visto que é predominantemente a mãe quem cuida de maneira mais próxima da criança desde as menores idades, exercendo assim importante influência na adoção de hábitos de vida saudáveis ou de risco à saúde entre seus filhos. 9

Sendo assim, cabe ressaltar que profissionais da área da saúde que trabalham diariamente com cuidados relacionados à saúde de crianças e adolescentes devem focar esforços na conscientização dos pais sobre seu importante papel no combate/ prevenção à obesidade abdominal entre os filhos. Além disso, esses profissionais devem, sempre que possível, estabelecer parcerias com o ambiente escolar, na tentativa de conscientizá-lo sobre seu importante papel nesse trabalho de prevenção.

Por fim, as principais limitações do presente estudo residem em seu delineamento transversal, característica esta que limita a inferência de relações de causalidade, e também o não controle de fatores de risco como atividade física e hábitos alimentares. Sendo assim, torna-se necessário indicar a importância da realização de futuros estudos que abordem essa questão em delineamentos longitudinais e englobem tais variáveis de confusão em seu protocolo de estudo. Adicionalmente, devido à ausência de valores críticos específicos para a população pediátrica brasileira, 27 ressalta-se a dificuldade em detectar a prevalência da obesidade abdominal, pois as prevalências observadas (mesmo elevadas) poderiam estar subestimadas, uma vez que os valores adotados são oriundos da população americana, ${ }^{17}$ que apresenta elevada ocorrência de S/O.1

Com base nos resultados observados é possível concluir que a prevalência de obesidade abdominal observada nos adolescentes analisados foi elevada, aproximando-se de $15 \%$. Além disso, as informações 
apresentadas permitem concluir que o meio escolar, o excesso de peso materno e de ambos os pais são fatores familiares que se associam com significativa magnitude à presença da obesidade abdominal entre adolescentes.

\section{Agradecimentos}

Os autores agradecem à Coordenação de Aperfeiçoamento do Pessoal de Nível Superior (CAPES)

\section{Referências}

1. Wang Y, Monteiro C, Popkin BM. Trends of obesity and underweight in older children and adolescents in the United States, Brazil, China, and Russia. Am J Clin Nutr. 2002; 75: 971-7.

2. Cintra IP, Passos MA, Fisberg M, Machado HC. Evolution of body mass index in two historical series of adolescents. $\mathrm{J}$ Pediatr (Rio J). 2007; 83: 157-62.

3. Ferreira AP, Oliveira CE, França NM. Metabolic syndrome and risk factors for cardiovascular disease in obese children: the relationship with insulin resistance (HOMA-IR). J Pediatr (Rio J). 2007; 83: 21-6.

4. Buff CG, Ramos E, Souza FI, Sarni RO. Frequency of metabolic syndrome in overweight and obese children and adolescents. Rev Paul Pediatr. 2007; 25: 221-6.

5. Rosa ML, Mesquita ET, da Rocha ER, Fonseca VM. Body mass index and waist circumference as markers of arterial hypertension in adolescents. Arq Bras Cardiol. 2007; 88: 573-8.

6. Sinaiko A. Obesity, insulin resistance and the metabolic syndrome. J Pediatr (Rio J). 2007; 83: 3-4.

7. Almeida CA, Pinho AP, Ricco RG, Elias CP. Abdominal circumference as an indicator of clinical and laboratory parameters associated with obesity in children and adolescents: comparison between two reference tables. J Pediatr (Rio J). 2007; 83: 181-5.

8. Neovius M, Linné Y, Rossner S. BMI, waist-circumference and waist-hip-ratio as diagnostic tests for fatness in adolescents. Int J Obes Relat Metab Disord. 2005; 29: 163-9.

9. Mendes MJ, Alves JG, Alves AV, Siqueira PP, Freire EF. Associação de fatores de risco para doenças cardiovasculares em adolescente e seus pais. Rev Bras Saude Matern Infant. 2006; 6: 49-54s.

10. Silveira D, Taddei JA, Escrivão MA, Oliveira FL, AnconaLopez F. Risk factors for overweight among Brazilian adolescents of low-income families: a case-control study. Public Health Nutr. 2006; 9: 421-8.

11. Suñé FR, Dias-da-Costa JS, Olinto MT, Pattussi MP. Prevalence of overweight and obesity and associated factors among schoolchildren in a southern Brazilian city. Cad Saude Publica. 2007; 23: 1361-71.

12. Terres NG, Pinheiro RT, Horta BL, Pinheiros KA, Hotra LL. Prevalence of factors associated to overweight and obesity in adolescents. Rev Saude Publica. 2006; 40: 1-6. pela bolsa de Mestrado cedida ao estudante Rômulo Araújo Fernandes durante o segundo semestre do ano de 2007.
13. Marins VM, Almeida RM, Pereira RA, Azevedo Barros MB. The relationship between parental nutritional status and overweight children/adolescents in Rio de Janeiro, Brazil. Public Health. 2004; 118: 43-9.

14. Fernandes RA, Rosa CS, Buonani C, Oliveira AR, Freitas Júnior IF. The use of bioelectrical impedance to detect excess visceral and subcutaneous fat. J Pediatr (Rio J). 2007; 83: 529-34.

15. Gordon CC, Chumlea WC, Roche AF. Stature, recumbent length and weight. In: Lohman TG, Roche AF, Martorel R, editors. Anthropometric standardization reference manual. Champaign: Human Kinetics Books; 1988. p. 3-8.

16. Callaway CW, Chumlea WC, Bouchard C, Himes JH, Lohman TG, Martin AD, Mitchell CD, Mueller WH, Roche AF, Seefeldt VD. Circunferences. In: Lohman TG, Roche AF, Martorel R, editors. Anthropometric standardization reference manual. Champaign: Human Kinetics Books; 1988. p. 39-54.

17. Taylor RW, Jones JE, Williams SM, Goulding A. Evaluation of waist circumference, waist-to-hip, and the conicity index as screening tools for high trunk fat mass, as measured by dual-energy X-ray absorptiometry, in children aged 3-19y. Am J Clin Nutr. 2000; 72: 490-5.

18. Instituto Brasileiro de Opinião Pública e Estatística. Levantamento socioeconômico -2000-IBOPE. Rio de Janeiro. [acesso em 2 fev 2003]. Disponível em: http://www.anep.org.br

19. Costa RF, Cintra IS, Fisberg M. Prevalência de sobrepeso e obesidade em escolares da cidade de Santos, SP. Arq Bras Endocrinol Metab. 2006; 50: 60-7.

20. Oliveira AM, Oliveira AC, Almeida MS, Oliveira N, Adan L. Influence of the family nucleus on obesity in children from northeastern Brazil: a cross-sectional study. BMC Public Health. 2007; 7: 235.

21. Campos LA, Leite AJ, Almeida PC. Prevalência de sobrepeso e obesidade em adolescentes escolares do município de Fortaleza, Brasil. Rev Bras Saude Matern Infant. 2007; 7: 183-90.

22. Nunes MMA, Figueiroa JN, Alves JG. Overweight, physical activity and foods habits in adolescents from different economic levels, Campina Grande (PB). Rev Assoc Med Bras. 2007; 53: 130-4. 
23. Veugelers PJ, Fitzgerald AL. Effectiveness of school programs in preventing childhood obesity: a multilevel comparison. Am J Public Health. 2005; 95: 432-5.

24. Fernandes RA, Junior IF, Cardoso JR, Vaz Ronque ER, Loch MR, Oliveira AR. Association between regular participation in sports and leisure time behaviors in Brazilian adolescents: a cross-sectional study. BMC Public Health. 2008; 8: 329.

25. Fernandes RA, Casonatto J, Christofaro DG, Ronque ER, Oliveira AR, Freitas Júnior IF. Riscos para o excesso de peso entre adolescentes de diferentes classes socioeconômicas. Rev Assoc Med Bras. 2008; 54:334-8.

Recebido em 28 de fevereiro de 2008

Versão final apresentada em 22 de setembro de 2009

Aprovado em 19 de outubro de 2009
26. Ortega FB, Ruiz JR, Sjostrom M. Physical activity, overweight and central adiposity in Swedish children and adolescents: the European Youth Heart Study. Int J Behav Nutr Phys Act. 2007; 4: 61.

27. Guimarães IC, de Almeida AM, Santos AS, Barbosa DB, Guimarães AC. Blood pressure: effect of body mass index and of waist circumference on adolescents. Arq Bras Cardiol. 2008; 90: 393-9 